\title{
INTRODUCTION OF CLOUDS IN DART MODEL
}

\author{
Y. Wang ${ }^{1, *}$, J. Grimaldi ${ }^{2}$, L. Landier ${ }^{3}$, E. Chavanon ${ }^{1}$, J. P. Gastellu-Etchegorry ${ }^{1}$ \\ ${ }^{1}$ CESBIO, Toulouse University, CNRS, CNES, IRD, PSU, Toulouse 31400, France - (wangy, jean-philippe.gastellu, \\ eric.chavanon)@cesbio.cnes.fr \\ ${ }^{2}$ INRAE UMR System, Montpellier 34000, France - juliette.grimaldi@inrae.fr \\ ${ }^{3}$ CNES (Centre National d'Etudes Spatiales), Av. E. Belin, Toulouse 31400, France - lucas.landier@cnes.fr
}

\section{Commission VI, WG VI/4}

KEY WORDS: Clouds, DART, Atmosphere, Radiative Transfer, Modelling, Inversion

\begin{abstract}
:
Clouds cover around two thirds of the Earth's surface. Most of them are thick enough to influence the radiative budget of our planet: they increase the top of atmosphere (TOA) exitance and they alter the bottom of atmosphere (BOA) direct and diffuse irradiance. However, most radiative transfer models dedicated to Earth surfaces, such as DART (Discrete Anisotropic Radiative Transfer), simulate only cloudless atmospheres. We recently introduced clouds in DART in order to improve the modelling of weather for remote sensing simulations. In this implementation, clouds were characterized with user specified optical properties and vertical distribution. They were modelled as layered one-dimensional medium that coexists with gases and aerosols. The atmospheric radiative transfer modelling relies on the discrete ordinate method already in DART. In addition, an iterative inversion procedure was designed to test this improvement with field measurements during two cloudy days at Lamasquère meteorological station (France). Specifically, it derives time-series of atmosphere parameters from time-series of BOA solar irradiance measurements. These inversed atmospheric parameters were used to simulate total and diffuse BOA irradiance in PAR (Photosynthetically Active Radiation) domain. The comparison of time-series of measured and DART simulated PAR irradiance lead to very encouraging results (mean relative error $8 \%$ for total irradiance and $\sim 20 \%$ for diffuse irradiance). It stresses the potential of DART to accurately simulate irradiance in cloudy days.
\end{abstract}

\section{INTRODUCTION}

Clouds are one of the most common weather phenomena that greatly influence optical remote sensing (RS) observations. Their global coverage with an optical depth larger than 0.1 is $\sim 68 \%$, with an optical depth larger than 2.0 is $\sim 56 \%$ (Stubenrauch et al. 2013). Most clouds are thick enough to strongly influence the top (TOA) and bottom (BOA) of atmosphere irradiance. For example, in presence of dense cloud cover, the BOA direct irradiance tends to be zero since the solar radiation is totally diffused. However, most Earth surface radiative transfer (RT) models, such as DART, LESS and RAPID, simulate only clear sky irradiance, which limits their use in practical applications. Recently, we designed a cloud modelling method in DART model for simulating accurate direct and diffuse irradiance in cloudy days. Clouds are simulated as layered one-dimensional medium that suspend in the atmosphere in order to adapt to current DART atmospheric RT modelling.

To assess its validity, we investigated the possibility to determine atmospheric parameters (i.e., aerosols and clouds optical thicknesses) such that DART simulated total BOA irradiance $E_{\text {BOA,DART }}\left(\Delta \lambda_{1}\right)$ over a given spectral interval $\Delta \lambda_{1}$ be equal to actual field measurements $E_{\mathrm{BOA} \text {,measure }}\left(\Delta \lambda_{1}\right)$. To achieve this objective, we designed a DART-based atmosphere inversion method. The determined atmospheric parameters can be applied to simulate total BOA irradiance $E_{\mathrm{BOA}, \mathrm{DART}}\left(\Delta \lambda_{2}\right)$ over any other spectral interval $\Delta \lambda_{2}$. The comparison between $E_{\mathrm{BOA}, \mathrm{DART}}\left(\Delta \lambda_{2}\right)$ and the field measurement $E_{\mathrm{BOA} \text {,measure }}\left(\Delta \lambda_{2}\right)$ at the same site is used to validate the cloud modelling.
This paper presents DART model, the new cloud modelling and the inversion method, and the validity assessment of cloud modelling using the inversion method and the field measurements.

\section{DART MODEL}

DART (https://dart.omp.eu) has been developed at CESBIO since 1992 (Gastellu-Etchegorry et al. 1996; Gastellu-Etchegorry et al. 2017). It is one of the most accurate and comprehensive three-dimensional RT models in the remote sensing community. It simulates the radiative budget, bi-directional reflectance factor (BRF) and images acquired by spectroradiometers at any altitude and along any viewing direction for 3D natural and urban scenes (i.e., landscapes), with topography and atmosphere (Figure 1), from visible to thermal infrared domain. For that, it uses an iterative discrete ordinate method (DOM) that tracks radiation fluxes along a finite number of discrete directions. It also simulates terrestrial and aero-spatial LiDAR signal (point cloud, waveform, photon counting) with an approach that combines Monte Carlo (MC) method and DOM.

DART simulates the atmosphere as the superimposition of three volumes: (1) the bottom atmosphere (BA) inside the simulated landscape, with voxels equal to the voxels of the landscape, (2) the mid-atmosphere (MA) made of layers or voxels, and (3) the high atmosphere (HA) made of layers. The possibility to have voxels per layer of the mid-atmosphere allows DART to simulate the spatial heterogeneity of the atmospheric backscattering. Any atmosphere layer/voxel is homogeneously filled with gases and

\footnotetext{
${ }^{*}$ Corresponding author
} 


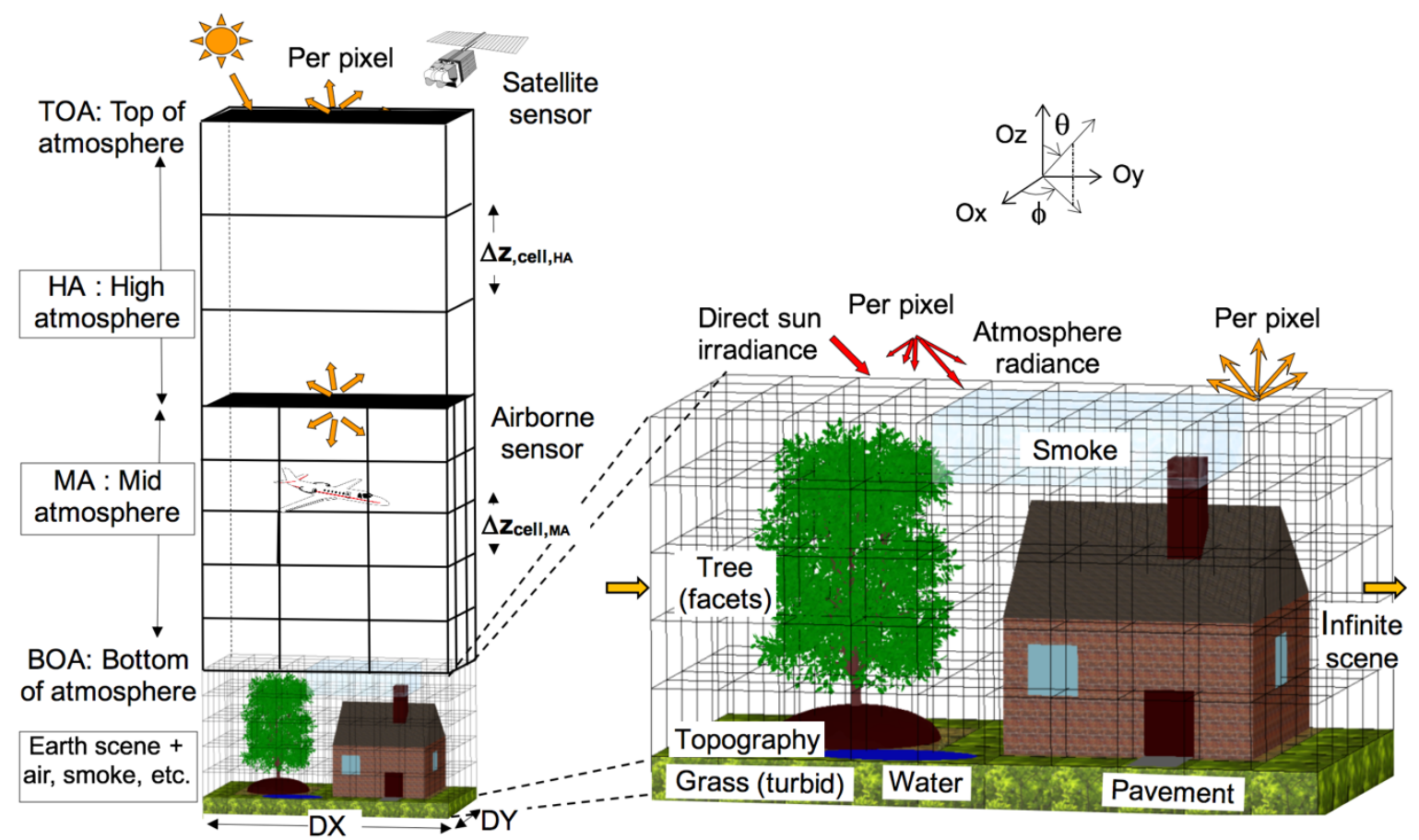

Figure 1. DART 3D discrete mock-up. Landscape elements are made of facets (triangles), and/or fluid and turbid vegetation voxels.

The simulated landscape is represented by a cell matrix in order to optimize ray tracing. The atmosphere is represented by three volumes: upper level (HA) made of layers, middle level (MA) made of voxels of any size, and lower level (BA) in the landscape cell matrix

aerosols that have specific physical (i.e., temperature, pressure, density) and spectral (i.e., absorption/scattering extinction coefficient, scattering phase function) properties. These physical and optical properties are either user-defined or computed from DART atmosphere SQL database that was initially derived from MODTRAN simulations and LOWTRAN source code. The atmospheric RT modelling relies on the DOM and the spectral application of Beer's law with band mean optical properties (Gastellu-Etchegorry et al. 2004). This modelling is capable to simulate the direct and diffuse irradiances at any altitude (TOA, $\mathrm{BOA}$, etc.) in the atmosphere as well as the radiative coupling with Earth surface RT modelling.

\section{CLOUD MODELLING AND INVERSION}

\subsection{DART cloud modelling}

Initially, DART atmospheric RT modelling was adapted to atmosphere made of aerosols and gases. The BOA and TOA irradiance was inexact during cloudy days. In order to better simulate actual irradiance in the presence of clouds, smog, etc., we introduced a new element "cloud" in the DART discrete atmosphere. In a first step, this "cloud" was modelled as layered one-dimensional medium rather than voxelized threedimensional medium since the layered medium is more suitable for current DART atmospheric RT modelling. Clouds are characterized by four properties: (1) a spectral vertical optical depth from the Earth surface to TOA, (2) a vertical density profile, (3) a single scattering albedo of its components, and (4) a scattering phase function. Typical spectral optical depth and single scattering albedo of two types of clouds (i.e., Cumulus and Stratus) are stored in the DART atmosphere SQL database. They were derived from MODTRAN simulations. Users can also define any specific cloud type.
This new functioning allows one or more types of clouds coexist with gases and aerosols in DART atmosphere. However, it is very inefficient to compute one-by-one constituent the multiple scattering. For that, a technique similar to Berk et al. 1998 was applied to accelerate the multiple scattering modelling. In short, an effective single scattering albedo and an effective phase function weighted by the scattering extinction coefficient are used to simulate the multiple scattering of the combined aerosols and clouds. An aerosol or a cloud is called "non-gas element" in the combined mixture. This technique gives the same scattered radiance as the combined scattered radiance computed one-byone element while it costs much less time.

Initial DART atmospheric RT modelling is detailed in Grau and Gastellu-Etchegorry, 2013. Here we present three major steps for the new cloud modelling. Without loss of generality, we consider the case of $n$ non-gas elements, for a single spectral band.

1) Preliminary data processing: band mean optical properties (i.e., vertical optical depth $\tau_{p, n}$, single scattering albedo $\omega_{n}$, parameters of double Henyey-Greenstein phase function) per element are computed from optical properties at $1 \mathrm{~cm}^{-1}$ spectral resolution in the DART atmosphere SQL database. Aerosol extinction coefficient profiles $\left(\alpha_{p}^{550 \mathrm{~nm}}(z)\right)$ at 550 $\mathrm{nm}$ and cloud density profiles $\left(\rho_{\text {cld }}(z)\right)$ are directly extracted from the DART atmosphere SQL database.

2) Computation of extinction coefficient and effective scattering albedo: DART atmosphere consists of $j$ discrete layers, with layer thickness $\Delta z_{j}$ per layer. The total extinction coefficient $\alpha_{n, j}$ per element $n$ per layer $j$ is $\alpha_{n, j}=\frac{\tau_{p, n}}{\Delta z_{j}}$. 
$\frac{\int_{\Delta z_{j}} \alpha_{p}^{550 \mathrm{~nm}}(z) d z}{\int_{0}^{\mathrm{TOA}} \alpha_{p}^{550 \mathrm{~nm}^{2}}(z) d z}$ for aerosols and $\alpha_{n, j}=\frac{\tau_{p, n}}{\Delta z_{j}} \cdot \frac{\int_{\Delta z_{j}} \rho_{\mathrm{cld}}(z) d z}{\int_{0}^{\mathrm{TOA}} \rho_{\mathrm{cld}}(z) d z}$ for clouds. The single scattering albedo $\omega_{n}$ per element $n$ is supposed to be independent of altitude. The fraction of scattering extinction coefficient $p_{n, j}$ and the effective single scattering albedo $\bar{\omega}_{j}$ are computed in a later step: $p_{n, j}=$ $\frac{\alpha_{n, j^{*}} \omega_{n, j}}{\sum_{n} \alpha_{n, j^{\prime}} \omega_{n, j}}$, with $\sum_{n} p_{n, j}=1$, and $\bar{\omega}_{j}=\frac{\sum_{n} \alpha_{n, j^{*}} \omega_{n, j}}{\sum_{n} \alpha_{n, j}}$.

3) Computation of effective scattering phase function: the scattering mechanism is modelled with one effective scattering phase function for all non-gas elements: $\bar{P}_{j}(\varphi)=$ $\sum_{n} p_{n, j} \cdot P_{n, j}(\varphi)$, with $P_{n, j}(\varphi)$ the scattering phase function of element $n$ at layer $j, \varphi$ is the scattering phase angle.

Note that this flexible implementation also makes it possible to simulate cloud-only atmosphere or aerosol-only atmosphere.

\subsection{Inversion procedure based on cloud modelling}

Time-series of BOA irradiance $E_{\mathrm{BOA} \text {,measure }}(t)$ is commonly measured in broad spectral bands by in-situ sensors (e.g., BF5: www.delta-t.co.uk/product/bf5). Hence, with the objectives to (1) assess the validity of the new DART cloud modelling and to (2) make use of the measured broadband data in practical applications, we investigated to simulate the time-series of BOA direct $E_{\mathrm{BOA}, \mathrm{dir}, \mathrm{DART}}(\lambda, t)$ and diffuse $E_{\mathrm{BOA}, \text { diff,DART }}(\lambda, t)$ irradiance for any spectral band $(\lambda, \Delta \lambda)$ with atmosphere parameters derived from $E_{\mathrm{BOA} \text {,measure }}(t)$ using a DART-based atmospheric inversion procedure (Figure 2). The detailed flowchart of the inversion procedure is in the Appendix.

This iterative inversion procedure assesses two main varying atmosphere parameters (i.e., aerosols and clouds optical depths $\Delta \tau_{\text {aerosol }}(\lambda, t)$ and $\left.\Delta \tau_{\text {cloud }}(\lambda, t)\right)$ per time step $t$ such that DART simulated broadband BOA irradiance $E_{\mathrm{BOA}, \mathrm{DART}}(t)$ is equal to $E_{\mathrm{BOA} \text {,measure }}(t) . \Delta \tau_{\text {aerosol }}(\lambda, t)$ and $\Delta \tau_{\text {cloud }}(\lambda, t)$ are adjusted by multiplying by specific factors $f$ the optical depths of the selected aerosol and cloud models. Note that gas state (i.e., optical properties, vertical distribution, etc.) is supposed to be constant each day.

$$
\begin{aligned}
& f_{\text {cloud }}(t)=f_{\text {aerosol }}(t)=0 \\
& \text { do }\{ \\
& \text { if }\left(f_{\text {aerosol }}(t) \leq f_{\text {aerosol,Max }}(t)\right)\{ \\
& E_{\mathrm{BOA}, \mathrm{DART}}(t)=\operatorname{DART} \operatorname{simulation}\left(f_{\text {aerosol }}(t), f_{\text {cloud }}(t)\right) \\
& f_{\text {aerosol }}(t)=\operatorname{adjust}\left(f_{\text {aerosol }}(t), E_{\mathrm{BOA}, \mathrm{DART}}(t), E_{\mathrm{BOA}, \text { measure }}(t)\right) \\
& \} \text { else }\{ \\
& E_{\mathrm{BOA}, \mathrm{DART}}(t)=\text { DART } \operatorname{simulation}\left(f_{\text {aerosol,Max }}(t), f_{\text {cloud }}(t)\right) \\
& f_{\text {cloud }}(t)=\operatorname{adjust}\left(f_{\text {cloud }}(t), E_{\mathrm{BOA}, \mathrm{DART}}(t), E_{\mathrm{BOA}, \text { measure }}(t)\right) \\
& \text { \} } \\
& \} \text { while }\left(\frac{\left|E_{\mathrm{BOA}, \mathrm{DART}}(t)-E_{\mathrm{BOA}, \text { measure }}(t)\right|}{E_{\mathrm{BOA}, \text { measure }}(t)}<\varepsilon\right)
\end{aligned}
$$

Figure 2. Pseudo code of the inversion algorithm that searches per time step $t$ for aerosol factor $f_{\text {aerosol }}(t)$ and cloud factor $f_{\text {cloud }}(t)$ such that $\frac{\left|E_{\mathrm{BOA}, \mathrm{DART}}(t)-E_{\mathrm{BOA}, \mathrm{measure}}(t)\right|}{E_{\mathrm{BOA}} \text { measure }(t)}<\varepsilon$, with $\varepsilon$ the relative error threshold. Step 1: cloudless assumption $\left(f_{\text {cloud }}(t)=0\right)$. The procedure iteratively searches for $f_{\text {aerosol }}(t)$. If this is not possible (i.e., maximum aerosol factor $f_{\text {aerosol,Max }}$ gives overestimated BOA irradiance), $f_{\text {cloud }}(t)$ is iteratively searched, using $f_{\text {aerosol }}(t)=f_{\text {aerosol,Max }}$ (default $f_{\text {aerosol,Max }}$ value $=1.0)$

\section{RESULTS AND DISCUSSION}

\subsection{Results}

The validation was conducted with instantaneous values of irradiance measured every 30 minutes of the day by a meteorological station located at Lamasquère $\left(43^{\circ} 29^{\prime} 47^{\prime} ' \mathrm{~N}\right.$, $1^{\circ} 14^{\prime} 16^{\prime} ' \mathrm{E}, 180-\mathrm{m}$ average elevation in Haute-Garonne department, France). We considered time-series of BOA irradiance in both solar $(0.4 \mu \mathrm{m}-2.7 \mu \mathrm{m})$ and PAR (Photosynthetically Active Radiation, $0.4 \mu \mathrm{m}-0.7 \mu \mathrm{m}$ ) domain acquired on July 14, 2016 and August 18, 2016. On July 14, 2016, the sky was cloudy several times while on August 18, 2016, the sky was totally cloudy. The PAR pyranometer (BF5 Sunshine Sensor, total irradiance accuracy $\pm 12 \%$, diffuse irradiance accuracy $\pm 15 \%$ ) recorded the photosynthetic photon flux density in $\mu \mathrm{mol} \cdot \mathrm{s}^{-1} \cdot \mathrm{m}^{-2}$. It was converted into an energy flux in $\mathrm{W} \cdot$ $\mathrm{m}^{-2}$ using the approximation $1 \mathrm{~W} \cdot \mathrm{m}^{-2}=4.57 \mu \mathrm{mol} \cdot \mathrm{s}^{-1}$. $\mathrm{m}^{-2}$ (Sager and McFarlane, 1997). The solar BOA irradiance was measured in $\mathrm{W} \cdot \mathrm{m}^{-2}$ by a SPN1 (www.deltat.co.uk/product/spn1) sunshine sensor (total and diffuse irradiance accuracy $\pm 8 \%$ )

The time-series of total irradiance in solar domain were used in the inversion procedure to derive atmosphere parameters. For that, DART simulations were configured with the MIDLATSUM (mid-latitude, $45^{\circ} \mathrm{N}$, July) standard atmosphere model, the Rural (visibility $23 \mathrm{~km}$ ) aerosol model. Clouds were configured with cumulus model with isotropic scattering phase function and homogeneous vertical profile between $3 \mathrm{~km}$ and $5 \mathrm{~km}$. Earth surface was configured with grass optical properties. The derived parameters were used to simulate BOA total and diffuse spectral irradiance in the PAR domain. The inversion was performed with two cases:

- Atmosphere without cloud modelling (i.e., atmosphere simulated with gases and aerosols only). In that case, aerosols act as a proxy of clouds, as previously tested by Grimaldi, 2018 . The decreases of BOA irradiance due to the passage of clouds is simulated by an increase of the aerosol optical depth.

- Atmosphere with cloud modelling (i.e., atmosphere simulated with gases, aerosols, and possibly clouds).

The comparison of the two cases aims to illustrate the interest of the new DART cloud modelling. Figure 3 show the measured and simulated BOA irradiance in solar and PAR domains every 30 minutes from 6:00 to 20:00 on the two dates, respectively. In both cases, DART solar irradiance is close to field measurement of solar irradiance since the inversion procedure aims to approach the measured solar irradiance: on July 14, 2016, Mean Relative Error $(\mathrm{MRE})=\frac{\sum_{t}\left|E_{\mathrm{DART}, t}-E_{\text {measure }, t}\right| / E_{\text {measure }, t}}{N}=7 \%$ without clouds, and MRE $=8 \%$ with clouds, with $N$ the number of measurements in a day (Figure 3.a, b); on August 18, 2016, MRE $=5 \%$ for both cases (Figure 3.c, d).

However, the simulated total and diffuse irradiance in PAR domain are much better with cloud modelling. On July 14, 2016, MRE decreases from $18 \%$ to $9 \%$ for total irradiance and decreases from $39 \%$ to $26 \%$ for diffuse irradiance. On August 18 , 2016, MRE decreases from $31 \%$ to $7 \%$ for total irradiance and decreases from $33 \%$ to $15 \%$ for diffuse irradiance. 

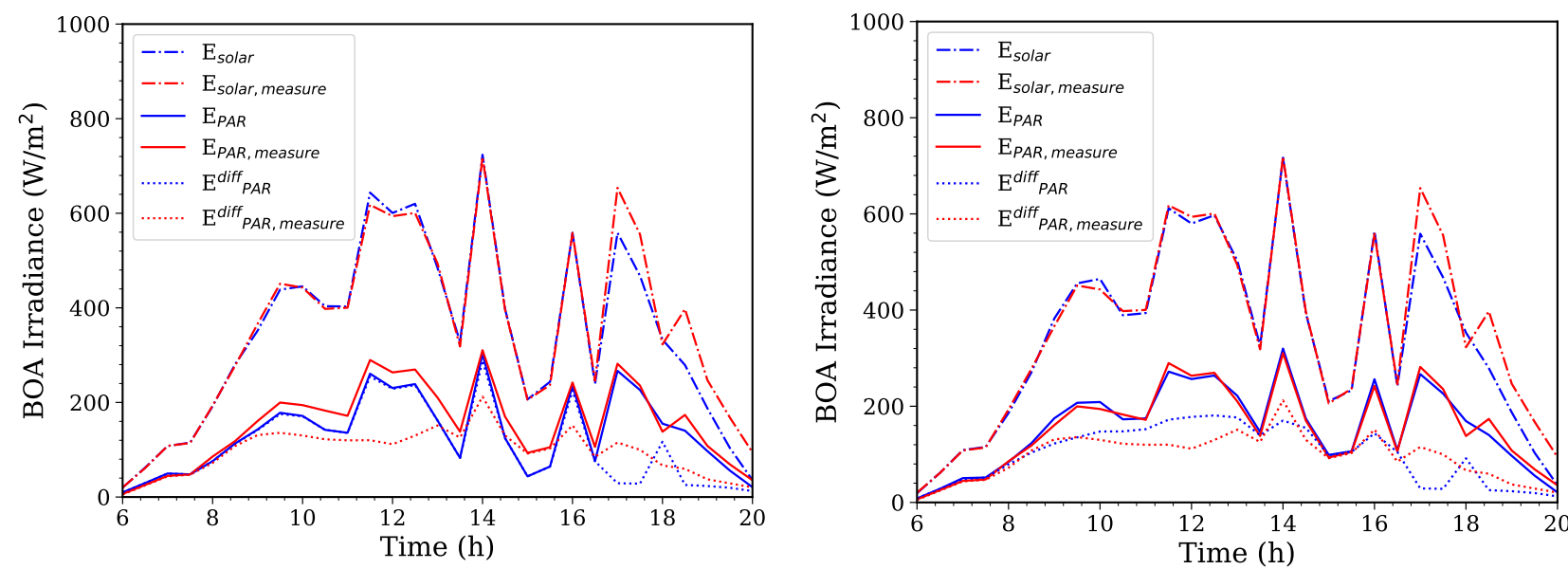

a)

b)
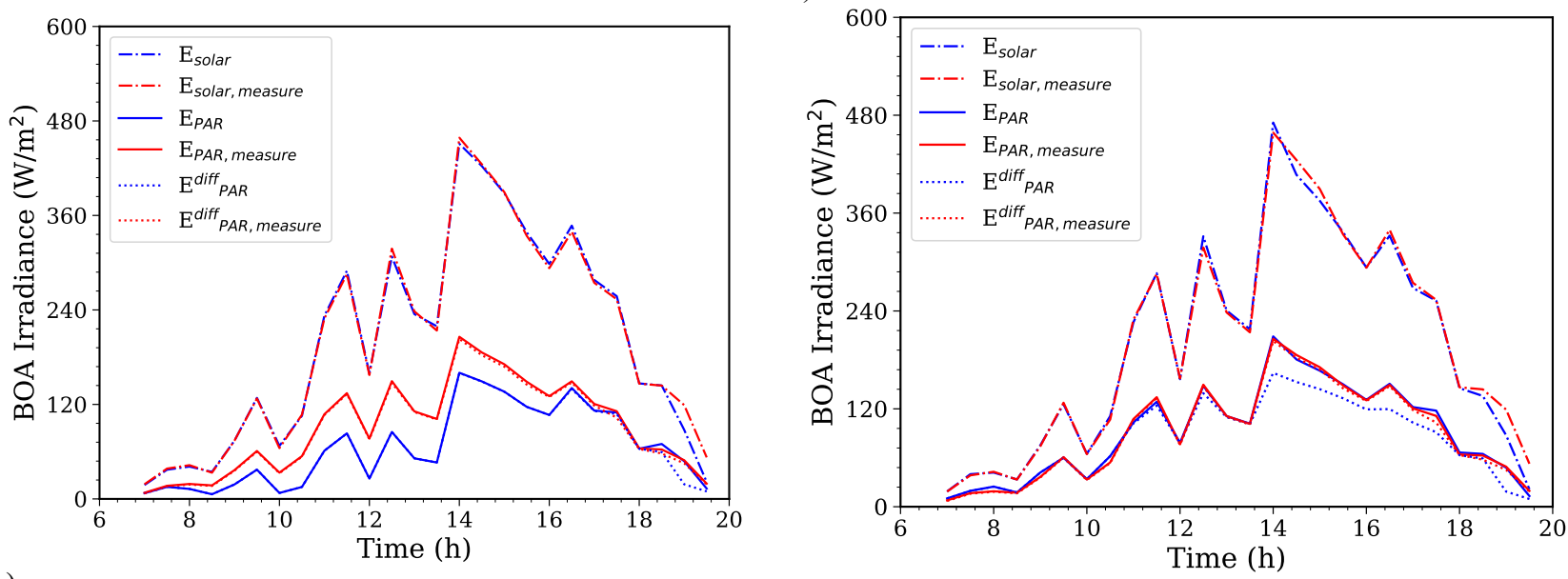

d)

Figure 3. Measured (red) and simulated (blue) BOA irradiance in solar and PAR spectral domains, on July 14, 2016 (a, b) and on August 18, 2016 (c, d). DART atmosphere is configured without clouds (a, c) and with clouds (b, d).

\subsection{Discussion}

In the "no cloud modelling" case, PAR total irradiance can be underestimated by as much as 50\% at 13:30 and the SKYL (ratio of diffuse and total irradiance) is almost $100 \%$ from 6:00 to $16: 00$ which is much larger than the measured SKYL (Figure 3.a). It illustrates that one cannot mimic the role of clouds with aerosols as in Grimaldi, 2018. Indeed, aerosol optical depth decreases exponentially with the wavelength, a change of aerosol optical influences much more the irradiance in visible domain than in infrared domain (green profile in Figure 4). On the other hand, cloud optical depth is more or less spectrally constant over the solar domain (Petty, 2006), a change of cloud optical depth influences equally the irradiance over the whole spectrum (blue profile in Figure 4). Therefore, in presence of actual clouds, in order to approach the actual solar irradiance, the inversion procedure with the option "no cloud" requires extreme aerosol optical depths (i.e., $f_{\text {aerosol }}(t)=38.5$ ), which in the PAR leads to greatly underestimated total irradiance and over-estimated SKYL. The new cloud modelling greatly improves this situation.

Although MRE of the simulated PAR total irradiance reaches the accuracy of pyranometers, the MRE of the simulated PAR diffuse irradiance still exceeds the pyranometer accuracy. It is probably due to the assumption of constant gas state during the inversion procedure. Indeed, water vapour is the major absorbing constituent in the solar domain, its molecule density varies with local temperature. As a result, the under- or over-estimated gas absorption gives over- or under-estimated diffuse irradiance.

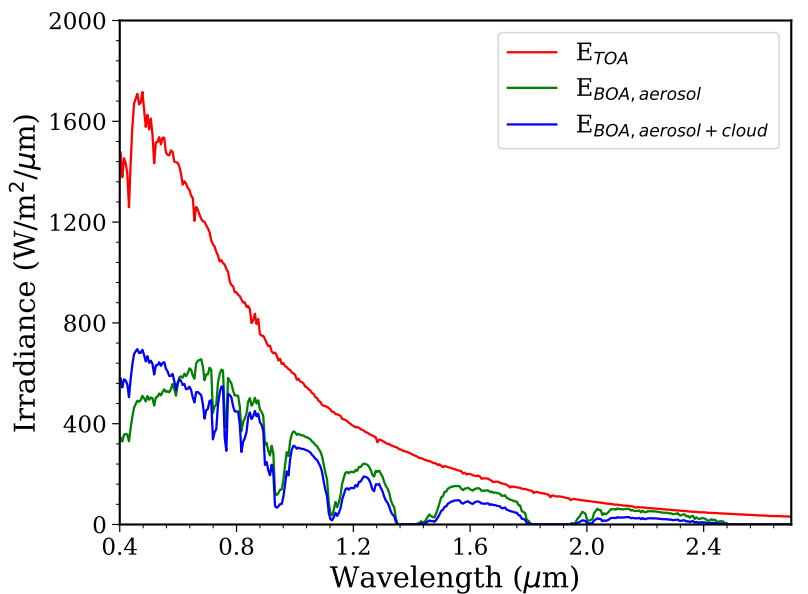

Figure 4. Hyperspectral (400 spectral bands) solar TOA irradiance (red) and BOA total irradiance simulated with clouds (blue) and no clouds (green) at 11:00. Atmosphere parameters are derived through the inversion procedure. 


\section{CONCLUDING REMARKS}

In this work, we introduced clouds in DART atmosphere and designed an inversion procedure to derive atmosphere parameters from in-situ measurements. Validation was conducted with the measurements from Lamasquère meteorological station. The comparison of time-series of measured and DART simulated PAR irradiance lead to very encouraging results MRE $\sim 8 \%$ for total irradiance and $\sim 20 \%$ for diffuse irradiance. It stresses the potential of DART to accurately simulate irradiance in cloudy days. Also, results stress that very large underestimate of irradiance in PAR domain, especially in blue domain occurs if aerosols are used to mimic clouds.

The inversion procedure proposed in this work can be potentially used for a wide range of experiment applications if only broadband BOA irradiance is available. Indeed, direct and diffuse BOA irradiance in narrow bands are commonly required for many urban and vegetation studies (Dissegna et al. 2019). This is typically the case for studying the sun induced chlorophyll fluorescence (Gastellu-Etchegorry et al. 2017).

\section{ACKNOWLEDGEMENTS}

This research is supported by the TOSCA program of CNES and the Occitanie region, France. We are grateful to the DART team computer scientists Dr. Nicolas Lauret and Jordan Guilleux for their support. Radiation measurements at Lamasquère are mainly supported by ICOS-France and the Regional Spatial Observatory (OSR) from CNRS, CNES and University of Toulouse. Regarding these data, we are also grateful to Franck Granouillac and Bartosz Zawilski for their technical support and Tiphaine Tallec and Aurore Brut for data processing.

\section{REFERENCES}

Berk, A., Bernstein, L.S., Anderson, G.P., Acharya, P.K., Robertson, D.C., Chetwynd, J.H. and Adler-Golden, S.M., 1998. MODTRAN cloud and multiple scattering upgrades with application to AVIRIS. Remote sensing of Environment, 65(3), 367-375.
Dissegna, M.A., Yin, T., Wei, S., Richards, D., Grêt-Regamey, A., 2019. 3-D reconstruction of an urban landscape to assess the influence of vegetation in the radiative budget. Forests, 10(8), 700.

Gastellu-Etchegorry, J.P., Demarez, V., Pinel, V., Zagolski, F., 1996. Modeling radiative transfer in heterogeneous 3-D vegetation canopies. Remote Sensing of Environment, 58(2), 131-56.

Gastellu-Etchegorry, J.P., Martin, E. and Gascon, F., 2004. DART: a 3D model for simulating satellite images and studying surface radiation budget. International Journal of Remote Sensing, 25(1), 73-96.

Gastellu-Etchegorry, J.P., Lauret, N., Yin, T., Landier, L., Kallel, A., Malenovský, Z., Al Bitar, A., Aval, J., Benhmida, S., Qi, J., Medjdoub, G., 2017. DART: recent advances in remote sensing data modeling with atmosphere, polarization, and chlorophyll fluorescence. IEEE Journal of Selected Topics in Applied Earth Observations and Remote Sensing, 10(6), 2640-2649.

Grimaldi J. 2018. Impacts of agroforestry on microclimate for grape and wine production: Assessment in Southern France. $\mathrm{PhD}$ thesis, Université Toulouse III Paul Sabatier.

Grau, E. and Gastellu-Etchegorry, J.P., 2013. Radiative transfer modeling in the Earth-Atmosphere system with DART model. Remote Sensing of Environment, 139, 149-170.

Petty, G.W., 2006: A first course in atmospheric radiation. Sundog Pub.

Sager, J.C. and McFarlane, J.C., 1997: Radiation. Plant growth chamber handbook, 1-29.

Stubenrauch, C.J., Rossow, W.B., Kinne, S., Ackerman, S., Cesana, G., Chepfer, H., Di Girolamo, L., Getzewich, B., Guignard, A., Heidinger, A., Maddux, B. C., 2013. Assessment of global cloud datasets from satellites: Project and database initiated by the GEWEX radiation panel. Bulletin of the American Meteorological Society, 94(7), 1031-1049. 


\section{APPENDIX}

Flowchart of the inversion python script in DART distribution.

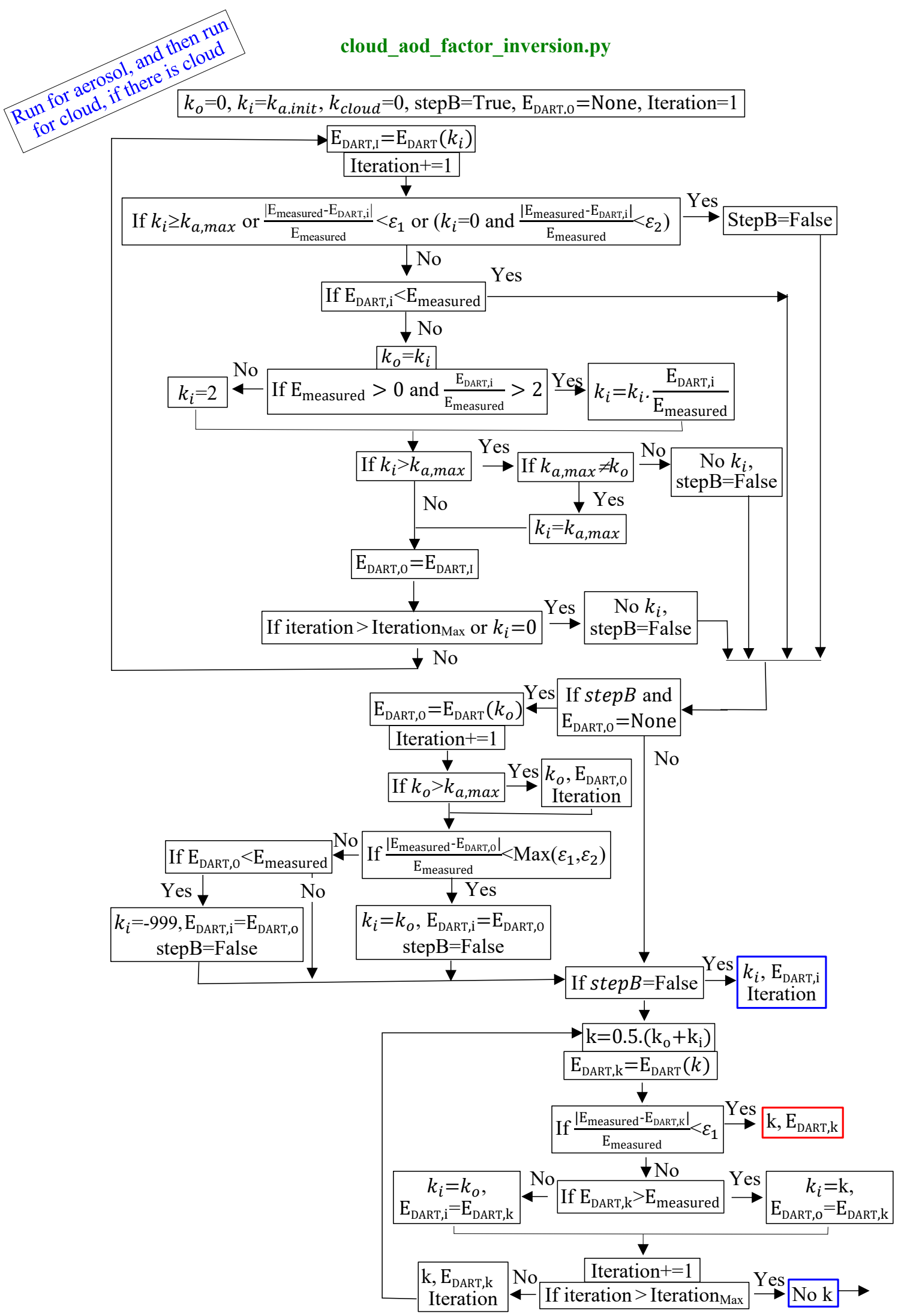

